\title{
Evaluation de la Dominance Temporelle des Sensations de vins rouges de Bourgogne et du Beaujolais par un panel d'experts et de consommateurs
}

\author{
Solène Brachet ${ }^{1}$, Mara Galmarini ${ }^{1,3}$, Bertrand Chatelet ${ }^{2}$, a, Anne-Laure Loiseau ${ }^{1}$, Sylvie Cordelle ${ }^{1}$, Valérie Lempereur $^{2}$ \\ et Pascal Schlich ${ }^{1}$ \\ ${ }^{1}$ INRA, Centre des Sciences du Goût et de l'Alimentation, Dijon, France \\ 2 Institut Français de la Vigne et du Vin (IFV) - SICAREX Beaujolais, 210 en Beaujolais, CS 60320, \\ 69661 Villefranche-sur-Saône Cedex, France \\ ${ }^{3}$ Consejo Nacional de Investigaciones Científicas y Técnicas (CONICET), Buenos Aires, Argentina
}

\begin{abstract}
Résumé. Deux groupes de 15 sujets ont été sélectionnés selon leur niveau d'expertise en dégustation de vin. Le premier groupe était constitué de professionnels du vin du Beaujolais habitués à réaliser du profil sensoriel, le second était constitué de consommateurs amateurs de vins rouges. Aucun n'avait expérimenté la méthode DTS (Dominance Temporelle des Sensations) auparavant. Six vins rouges ont été sélectionnés pour leur diversité sensorielle : trois vins du Beaujolais et trois de Bourgogne. Durant deux séances d'entraînement, 10 descripteurs (sucré-gras, amer, acide, astringent, fruits confits-cuits, fruits rouges, floral, végétal, épicé, et boisé) ont été définis et expliqués aux deux panels, qui ont aussi pu se familiariser avec le principe du DTS. A chacune des quatre séances suivantes, chaque panel a réalisé un profil DTS de chacun des six vins de l'étude. Les résultats montrent que les séquences de dominances des experts sont différentes de celles des consommateurs, soulignant l'importance de faire appel à des consommateurs pour la description de leur perception lorsque l'on souhaite comprendre leurs préférences. Les experts sont toutefois plus consensuels entre eux, ce qui se traduit par une meilleure discrimination des six vins. Cependant, la répétabilité individuelle, estimée grâce aux quatre répétitions, est légèrement meilleure pour les consommateurs que pour les experts, ce qui laisse envisager une utilisation prometteuse du DTS avec les consommateurs.
\end{abstract}

\section{Introduction}

La perception des sensations liées à la dégustation d'un aliment ou d'une boisson (vins) est un phénomène dynamique. Ainsi, plusieurs méthodes sensorielles ont été développées afin de prendre en compte cette notion de temporalité des sensations. La technique de Temps-Intensité (T-I) [1,2], le Dual Attribute TimeIntensity ou DATI [3], le Profil Progressif [4], la Dominance Temporelle des Sensations (DTS) [5,6], le Profil Séquentiel [7] montrent que la dimension temporelle peut être incluse dans l'évaluation sensorielle.

Toutes ces techniques mentionnées présentent des avantages et des inconvénients, mais la seule qui permet l'évaluation de plusieurs descripteurs simultanément et dynamiquement est le DTS. En effet, le T-I ne traite qu'un seul descripteur à la fois, alors que les méthodes de profil progressif ou séquentiel ne prennent en compte que partiellement la dynamique temporelle de la perception [8]. Avec le DTS, les panélistes sélectionnent parmi une liste de descripteurs préalablement définie, le descripteur qui retient le plus leur attention à un instant donné (défini comme étant le descripteur dominant) et réalisent cette

\footnotetext{
${ }^{a}$ Auteur de correspondance : bertrand. chatelet@ vignevin.com
}

tâche, à chaque moment et durant toute la durée de la dégustation. Alors que l'on trouve dans la littérature scientifique une soixante de références d'utilisation du DTS, seulement trois d'entre elles font référence aux vins [9-11]. Toutes ces études ont été, par ailleurs, conduites avec des sujets entraînés.

Pourtant, grâce à cette notion de dominance et à l'absence de valeur quantitative, l'évaluation devient presque instinctive, nécessitant moins d'entraînement. Dans ce cas le DTS pourrait, par voie de conséquence, être étendu aux consommateurs. A cet égard, l'hypothèse que les consommateurs soient capables de décrire les produits est de plus en plus acceptée au sein de la communauté scientifique d'analyse sensorielle [12]. D'autre part, ces derniers deviennent plus demandeurs et conscients des caractéristiques souhaitées dans certains produits, ce qui conduit à analyser leur perception avec attention [13] non seulement pour être en mesure de répondre à leurs attentes, mais également afin qu'ils puissent s'identifier au produit au moment de la communication réalisée sur celui-ci.

Ainsi, l'objectif de ce travail était de comparer les profils temporels DTS obtenus par un panel d'experts et un panel de consommateurs pour six vins de deux régions françaises, avec analyse de la répétabilité et de la discrimination. 
Tableau 1. Descripteurs du vin et références olfactives utilisées.

\begin{tabular}{|c|c|c|c|}
\hline $\begin{array}{l}\text { Type de } \\
\text { descripteur }\end{array}$ & Descripteur & Définition & Référence \\
\hline Texture & Astringent & $\begin{array}{l}\text { Sensation de rugosité sur } \\
\text { les muqueuses. }\end{array}$ & Pépins et peau de raisin. \\
\hline Saveur & Amer & $\begin{array}{l}\text { Correspond à la saveur } \\
\text { perçue avec une solution } \\
\text { de caféine }\end{array}$ & Endives, chocolat noir. \\
\hline Saveur & Acide & $\begin{array}{l}\text { Se dit d'une saveur aigre } \\
\text { et piquante }\end{array}$ & Jus de citron. \\
\hline Saveur & Sucré-gras & $\begin{array}{l}\text { Sucré = douceur d'un } \\
\text { vin Gras = donne la } \\
\text { consistance et viscosité }\end{array}$ & Solution de saccharose. \\
\hline Arôme & Épicé & $\begin{array}{l}\text { Arômes rappelant les } \\
\text { épices. }\end{array}$ & $\begin{array}{l}\text { Muscade, poivre, clous de } \\
\text { girofle. }\end{array}$ \\
\hline Arôme & Végétal & $\begin{array}{l}\text { Désigne une large } \\
\text { famille d'arômes. }\end{array}$ & $\begin{array}{l}\text { Herbe coupée (cis-3- } \\
\text { hexanol). }\end{array}$ \\
\hline Arôme & Boisé & $\begin{array}{l}\text { Marqué par les odeurs } \\
\text { apportées par l'élevage. }\end{array}$ & $\begin{array}{l}\text { Copeaux de bois, fût de } \\
\text { chêne. }\end{array}$ \\
\hline Arôme & Floral & $\begin{array}{l}\text { Arôme rappelant un } \\
\text { parfum de fleur. }\end{array}$ & Fleurs (violettes). \\
\hline Arôme & $\begin{array}{l}\text { Fruit confits- } \\
\text { cuits }\end{array}$ & $\begin{array}{l}\text { Arômes de fruits confi- } \\
\text { turés, confits. }\end{array}$ & $\begin{array}{l}\text { Arômes confiturés (confi- } \\
\text { ture de pruneaux). }\end{array}$ \\
\hline Arôme & Fruit rouges & $\begin{array}{l}\text { Désigne les parfums de } \\
\text { fruits rouges. }\end{array}$ & $\begin{array}{l}\text { Arôme fraise, framboise, } \\
\text { mûre, cassis. }\end{array}$ \\
\hline
\end{tabular}

\section{Matériels and méthode}

\subsection{Produits évalués}

Six vins ont été choisis dans deux régions de France, avec des caractéristiques sensorielles différentes : trois vins provenaient du Beaujolais (V2, V3, V4) et trois de Bourgogne (V1, V5, V6). Ces vins ont été fournis par le Bureau Interprofessionnel des Vins de Bourgogne (BIVB, Beaune, France) et l'Institut Français de la vigne et du Vin (IFV-SICAREX Beaujolais, Villefranche-sur-Saône, France).

\subsection{Procédure d'évaluation}

Les six vins ont été évalués par la méthode de la Dominance Temporelle des Sensations (DTS) [5]. Les panélistes avaient une liste de 10 descripteurs, chacun défini soit littéralement, soit par une référence physique (Tableau 1). Cette liste avait été choisie par les expérimentateurs en tenant compte des caractéristiques les plus pertinentes dans la littérature pour décrire les vins de ces régions et après dégustation des 6 vins de l'étude.

Chaque panéliste avait pour consigne de cliquer sur le descripteur qu'il percevait comme dominant, c'est-à-dire celui qui retenait le plus son attention, à chaque instant de la dégustation. Les données étaient collectées et analysées avec le logiciel internet TimeSens (www.timesens.com).

Pour se familiariser avec la méthode, les panélistes devaient suivre une séance d'entraînement. Pour les consommateurs, cette séance a aussi été utilisée pour les familiariser aux descripteurs du vin. Après l'entraînement, quatre séances d'évaluation ont alors été mises en place (d'une durée de 45 minutes chacune).
L'enregistrement des mesures commençait lorsque le panéliste cliquait sur le bouton «démarrer» simultanément à la mise en bouche du produit (une seule gorgée standardisée de $10 \mathrm{ml}$ ). Afin que l'évaluation soit proche de la consommation courante d'un vin, les panélistes avalaient le produit et continuaient à le décrire une fois dégluti. Lorsqu'ils ne percevaient plus aucune sensation, les panélistes devaient cliquer sur le bouton «arrêter» et l'enregistrement des mesures s'arrêtait. Les panélistes n'avaient aucune contrainte quant à la durée de dégustation du produit. Ils étaient libres de choisir plusieurs fois le même attribut comme dominant à des temps différents de la dégustation, ou au contraire de ne pas du tout sélectionner certains attributs s'ils ne les percevaient jamais comme dominants. Entre chaque produit, une pause de 30 secondes était imposée afin que les panélistes se rincent la bouche avec du pain frais et de l'eau.

Chaque panéliste avait un ordre de présentation des produits différent, généré selon un carré latin de Williams, et l'ordre de présentation des descripteurs sur l'écran était différent pour chaque personne, mais identique pour un même panéliste sur l'ensemble des vins. Les échantillons étaient servis dans des verres noirs munis de couvercles et identifiés par un code aléatoire à 3 chiffres et la température de la salle de dégustation était de $21^{\circ} \mathrm{C}$.

\subsection{Les panels d'évaluation}

Afin de pouvoir comparer deux panels distincts 15 panélistes ont été recrutés sur deux sites différents.

\subsubsection{Le panel d'experts}

Ce panel était constitué de cinq femmes et 10 hommes, âgés de 32 à 77 ans, habitant tous aux alentours de 
Villefranche-sur-Saône. Ils étaient tous des professionnels du vin habitués à déguster du vin pour différentes études sensorielles. Par contre, ils n'avaient jamais utilisé la méthode DTS.

\subsubsection{Le panel de consommateurs}

Ce panel était formé de 12 femmes et trois hommes, âgés de 20 à 70 ans. Ces consommateurs étaient des œnophiles mais non professionnels du vin. Ils ont été recrutés selon l'appartenance à l'un ou plusieurs des critères suivants: fréquence de consommation de vin rouge, lieu d'achat du vin, nombre de bouteilles de vins possédées en cave, abonnement éventuel à une revue sur le vin appartenance éventuelle à un club de dégustateurs de vin et ils devaient ne pas avoir fait de DTS auparavant.

\subsection{Analyses des données}

Les analyses des données DTS ont été faites en standardisant les durées de dégustation, qui n'étaient pas homogènes [14]. Cette standardisation permet d'obtenir plus de concordance entre les différents panélistes.

Afin de représenter les profils temporels, deux types de graphiques ont été réalisés : les courbes DTS et les bandplots. Les courbes DTS montrent, pour chaque échantillon, le taux de dominance pour chaque descripteur en fonction du temps $[5,6]$. Lorsque l'on obtient des courbes DTS associées à différents panels, il est possible de comparer facilement ces panels en soustrayant ces courbes une à une et en traçant des courbes de différences [5]. Les bandsplots représentent la compilation de toutes les données des panélistes pour un panel et un produit et chaque couleur représente un descripteur. Dans le band-plot d'un produit, un descripteur apparait seulement pour les périodes durant lesquelles son taux de dominance était significativement $(p=0,10)$ supérieur au taux attendu au hasard ; il apparait alors sous la forme de rectangles d'une couleur spécifique [15].

Afin de représenter graphiquement la discrimination des produits, une analyse en variables canoniques (CVA) des durées de dominance (temps durant lequel un descripteur a été cité comme dominant) a été effectuée pour chaque panel. La discrimination entre les produits apportée par cette analyse est évaluée par une statistique de Fisher qui permet de comparer les deux panels en termes de différences entre les produits. D'autre part, un test de dimensionnalité (nombre d'axes nécessaires pour représenter les différences entre les produits) permet de comparer les deux panels en termes de complexité de la discrimination entre les produits.

L'index de répétabilité des citations (CRI) a été calculé pour chaque panéliste, puis sur l'ensemble du panel afin de savoir si les sujets de l'un des deux panels étaient plus répétables que ceux de l'autre. Le CRI est défini pour chaque triplet (paneliste, produit, descripteur) par la formule:

$$
\mathbf{C R I}=|\mathbf{c}-\mathbf{r} / \mathbf{2}| /(\mathbf{r} / \mathbf{2})
$$

où $\mathrm{r}$ est le nombre de répétitions et $\mathrm{c}$ le nombre de répétitions pour lesquelles le panéliste a cité le descripteur comme dominant dans ce produit. La valeur du CRI est comprise entre 0 et 1 et est d'autant plus grande que la

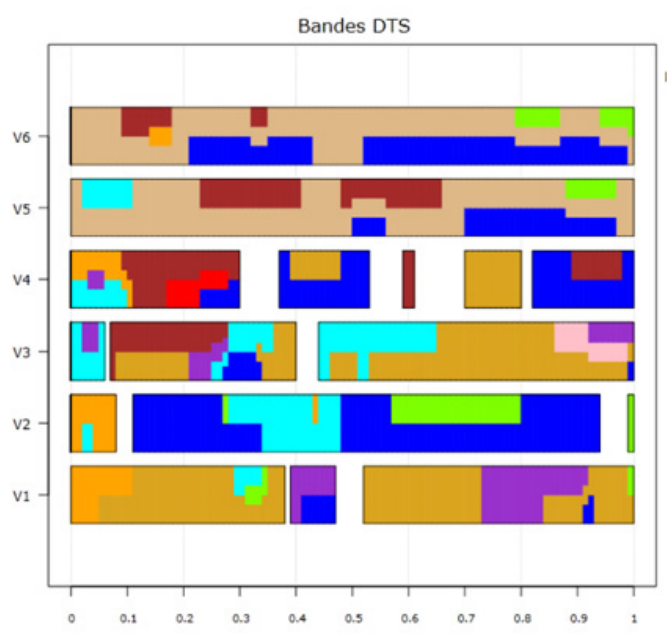

a)

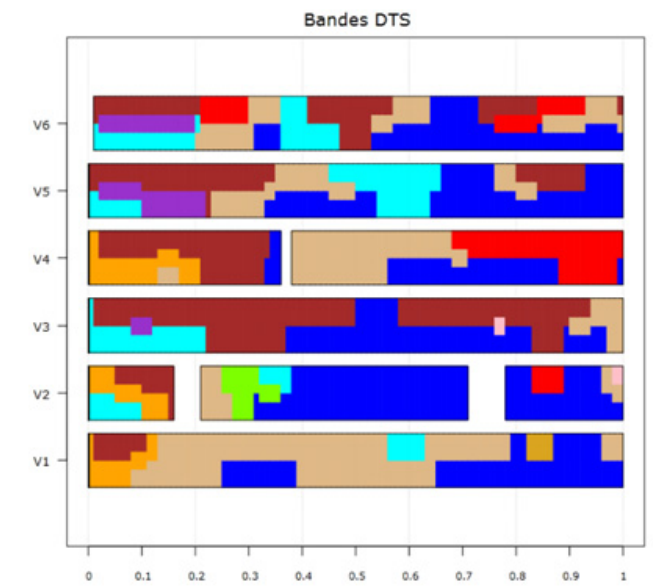

b)

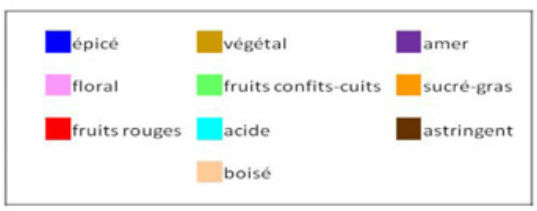

Figure 1. Band-plots standardisés. (a) panel de professionnels du vin et (b) panel de consommateurs.

répétabilité est bonne. Pour comparer les sujets d'un même panel, on calcule la moyenne des CRI de chaque sujet. Un CRI inférieur à 0,5 indique un problème de répétabilité du panéliste. Pour comparer les deux panels, on calcule les moyennes des CRI des sujets par panel.

\section{Résultats et discussion}

\subsection{Profils temporels des vins}

Les band-plots pour les profils temporels des six vins pour les deux panels sont représentés dans la Fig. 1 a et b. Lorsqu'il y a différentes couleurs pour un temps donné, cela signifie que plusieurs descripteurs ont été trouvés dominants à cet instant. A l'inverse, l'absence de couleur démontre qu'aucun descripteur n'a été jugé dominant à ce moment. Même si de manière générale les deux panels n'ont pas décrit les vins de la même façon, des similitudes peuvent être établies. 
Une attaque sucré-gras, suivie de l'arôme végétal puis de l'amertume alternée avec la dominance des notes épicées caractérisent le vin V1 selon les experts. Pour le même vin, si les consommateurs ont également perçu un début sucré-gras, ils ont, par ailleurs, cité comme dominants les arômes boisés et épicés. Pour le vin V2 les deux panels ont été davantage en accord. Ils ont, conjointement, décrit ce vin avec une dominance du caractère épicé accompagné d'arômes fruits confits-cuits et d'une certaine acidité. Pour le troisième vin (V3), l'attaque est acide et des caractéristiques d'astringence ont été relevées par les deux panels, de manière toutefois plus importante chez les consommateurs. Au niveau aromatique, pour les experts, le végétal constitue l'arôme principal du vin V3 alors que pour les consommateurs ce dernier a été dominé par des arômes épicés. Le vin V4 a été peut-être, pour les experts, l'échantillon le plus complexe à décrire, où la dominance a été partagée entre différents attributs : acide, sucré-gras (en attaque), astringent, fruits confits-cuits, épicé et végétal. Les consommateurs ont également défini ce vin avec une attaque sucré-gras mais, avec plus d'astringence et n'ont utilisé que les descripteurs épicé, boisé et fruits confits-cuits comme dominants. Enfin, les vins V5 et V6 ont été décrits par les experts comme étant dominés par le caractère boisé avec une certaine astringence, alors que chez les consommateurs, ces deux vins ont des profils temporels similaires: astringents, amers et acides en début de bouche, puis acides, épicés et boisés. Des notes fruits rouges ont, de plus, été perçues dans le vin V6.

Les experts ont plus fréquemment utilisé le descripteur « végétal » pour décrire les vins V1, V3 et V4 par rapport aux consommateurs. Ainsi, soit ce terme n'était pas suffisamment familier pour ces derniers, soit ils ont estimé qu'ils n'étaient pas le plus dominant pour eux. De même, les vins V5 et V6 sont davantage dominés par le caractère boisé chez les experts que chez les consommateurs.

Ces experts, issu d'un panel travaillant avec les vins du Beaujolais, sont plus habitués à déguster des vins de leur région plutôt que des vins de Bourgogne. Ce qui pourrait expliquer que lorsqu'on leur présente des vins de Bourgogne avec un caractère boisé, cette sensation domine toutes les autres, et qu'il leur est alors difficile de citer d'autres descripteurs pour caractériser ces vins. A l'inverse, chez les consommateurs bourguignons, plus habitués à déguster des vins de Bourgogne, le caractère boisé n'apparaît pas avec autant de dominance. Cette hypothèse va dans le sens de Saenz-Navajas et al. [16] qui ont démontré que la qualité intrinsèque d'un vin est perçue en lien avec la familiarité des panélistes pour ce vin. Le descripteur végétal a été peu perçu par les consommateurs (V3, V4) ou semble avoir été confondu avec le boisé (V1). En effet, Tu et al. [17], ont montré que deux panels de cultures différentes (Français et Vietnamien), sont consensuels en ce qui concerne les saveurs et les attributs de texture mais diffèrent par leurs différences culturelles pour la description des attributs liés aux arômes. Ce résultat n'est pas surprenant : en effet, la plupart du temps, les descripteurs qui distinguent deux types de panels sont liés aux arômes. Ce sont les descripteurs les plus difficiles à évaluer. Cette observation peut être expliquée par le fait

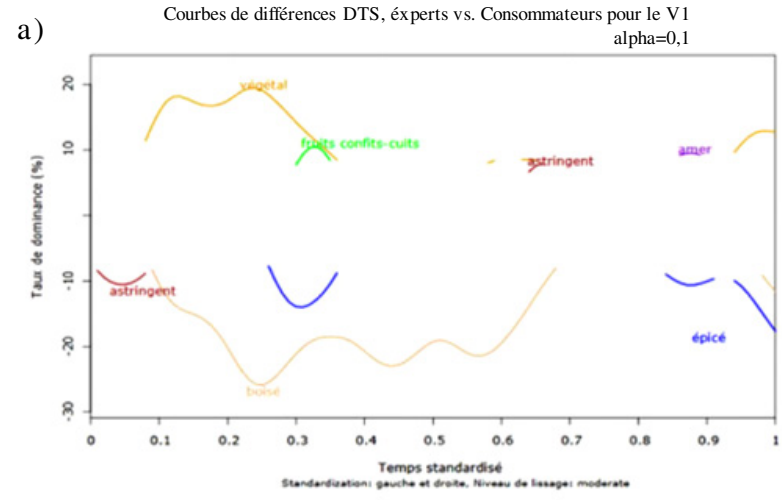

b) Courbes de différences DTS, éxperts vs. Consommateurs pour le V2

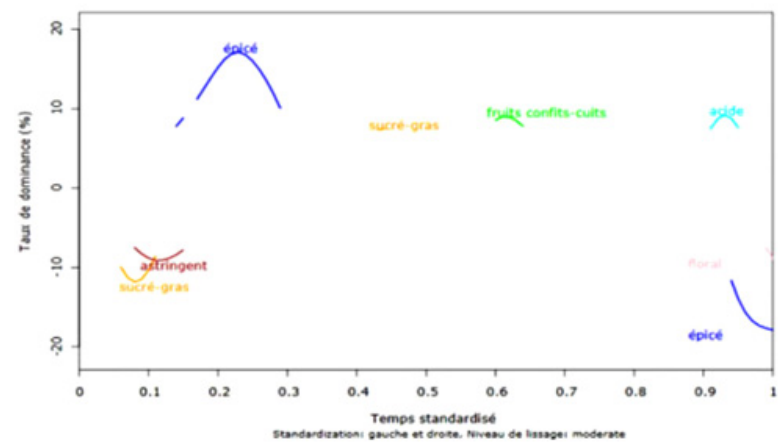

Figure 2. Courbes de différences DTS entre le panel d'éxperts (au dessus) et le panel de consommateurs (en dessous) pour les vins : a) V1 et b) V2.

que les descripteurs boisé et végétal sont sensoriellement proches. De plus, le descripteur végétal a pu sembler abstrait pour les consommateurs, alors qu'il s'agit d'un terme régulièrement employé par les experts du vin. Cette idée est confortée par le travail de Valentin et al. [18] qui montre que les experts du vin décrivent plus précisément les produits, utilisant à priori un vocabulaire normé. Les experts n'auraient pas de difficulté à distinguer ce type de descripteurs. Par contre la tâche semble plus compliquée pour les consommateurs qui ont moins l'habitude de décrire aussi précisément les vins. Pour cette raison, il est nécessaire de trouver des descripteurs qui soient explicites et compréhensibles pour les consommateurs.

La Fig. 2 a et b illustre les courbes de différence entre les deux panels. Si la figure représente peu de portions de courbes apparentes c'est donc qu'il existe peu de différences de description du vin entre les deux panels et on peut alors considérer que les panels sont en accord. Cette comparaison peut être faite pour chaque vin testé, seulement deux exemples sont présentés ci-dessous. Ainsi, la Fig. 2a montre qu'il existe d'importantes différences entre la description pour les deux panels pour le vin V1. En effet, les experts ont indiqué que ce vin avait un caractère végétal très présent au début de la dégustation alors que les consommateurs ont précisé que ce vin avait un caractère boisé très présent et qui perdure au cours du temps.

Par contre, pour le V2 (Fig. 2b), la perception est relativement similaire dans les deux groupes puisque peu de portions de courbes de différences sont significatives (tracées). Il semblerait juste que le caractère épicé de ce 
a)

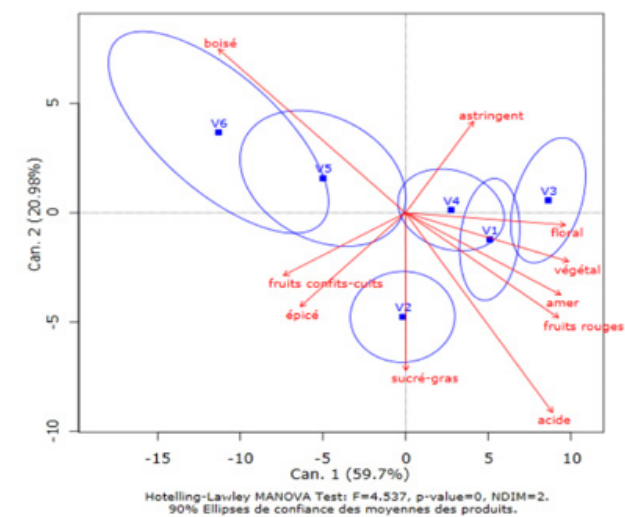

b)

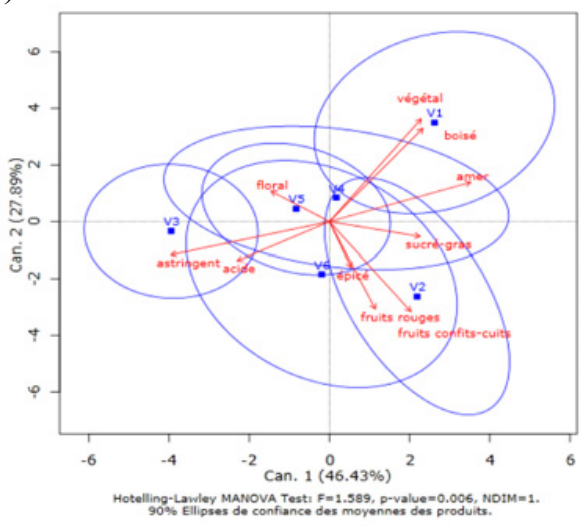

Figure 3. Analyse en variables canoniques (CVA) représentant la discrimination entre les 6 vins chez les experts (a) et chez les consommateurs (b) pour les 4 séances de mesure.

vin soit davantage perçu en début de bouche par le panel d'experts. Les autres portions de courbes dessinées sont trop petites pour signifier de réelles différences entre les panels.

\subsection{Discrimination des vins}

La Fig. 3 a et $b$ montre les CVA de chacun des deux panels. Cette représentation nous permet de savoir si les produits ont été différenciés entre eux par les durées de dominance. L'intersection entre les ellipses nous donne des informations quant aux différences entre les produits. Si les ellipses se chevauchent, c'est qu'à priori il n'existe pas de différence entre les produits, à l'inverse si les ellipses sont bien séparées, cela signifie qu'il existe une différence. Chaque descripteur est représenté par un vecteur indiquant sa direction croissante et dont la longueur est proportionnelle à la discrimination entre produits apportée par ce descripteur.

En comparant ces deux figures (Fig. 3 a et b), il est possible de voir très clairement que les résultats sont bien différents pour les deux panels. En effet, la statistique de test de Fischer F pour les experts est de 4,537 et deux dimensions significativement discriminantes. De plus, les ellipses de confiance sont petites et se chevauchent très peu, les produits peuvent donc être considérés comme différents. A l'inverse, la statistique de test chez les consommateurs n'est que de 1,589 et seule une dimension
Tableau 2. Regroupement des valeurs moyennes et extrêmes des CRI calculées pour les deux panels.

\begin{tabular}{|l|l|l|l|l|}
\hline Produit & Panel & Moyenne & Minimum & Maximum \\
\hline \multirow{2}{*}{ Vins } & Experts & 0,65 & 0,47 & 0,76 \\
\cline { 2 - 5 } & Consommateurs & 0,70 & 0,61 & 0,77 \\
\hline
\end{tabular}

est discriminante. Les ellipses de confiance pour les consommateurs sont plus grandes, il y a donc moins de séparation des produits que pour les experts. Cependant, les consommateurs ont quand même réussi à discriminer des vins. Ils ont différencié V1 et V3 mais aussi V2 et V3. Néanmoins, les experts restent toujours plus discriminants sur la base des durées de dominance que les consommateurs. Cette idée est tout à fait cohérente puisqu'ils connaissent beaucoup mieux le milieu du vin que les consommateurs.

\subsection{Répétabilité des panelistes}

Le Tableau 2 montre les valeurs moyennes, minimales et maximales des CRI individuels dans chacun des deux panels. Il existe au moins un panéliste chez les experts qui n'est pas répétable au fil des séances, tandis que les consommateurs n'ont pas obtenu de mauvaises valeurs de répétabilité individuelle. En ce qui concerne les moyennes sur les panels, on peut voir que le panel de consommateurs est légèrement plus répétable que le panel d'experts. Cette observation n'est pas liée au nombre de descripteurs utilisés pour chaque panel puisque la valeur médiane est environ la même pour les deux.

\section{Conclusions}

Les résultats obtenus lors cette expérimentation suggèrent que les séquences de dominances pour les experts et pour les consommateurs sont différentes. Il paraît donc important de prendre en compte la description de la perception des consommateurs vis-à-vis des différents produits testés, afin de mieux comprendre ultérieurement leurs préférences. Les experts s'avèrent plus consensuels entre eux et, de ce fait, offrent une meilleure discrimination des six vins. Cependant, la répétabilité individuelle, calculée grâce aux quatre séances de mesure, est légèrement meilleure pour les consommateurs que pour les experts, ce qui laisse envisager une utilisation prometteuse du DTS avec les consommateurs.

\section{Références}

[1] Lee III, W. and M. Pangborn, Time-intensity: the temporal aspects of sensory perception. Food Technology, 1986. 40(71): p. 78-82

[2] Neilson, A.J., Time-intensity studies. Drug and cosmetic Industry, 1957. 80(4): p. 452-3, 534

[3] Duizer, L.M., K. Bloom, and C.J. Findlay, Dualattribute time-intensity sensory evaluation: A new method for temporal measurement of sensory perceptions. Food Quality and Preference, 1997. 8(4): p. 261-269 
[4] Jack, F.R., J.R. Piggott, and A. Paterson, Analysis of Textural Changes in Hard Cheese during Mastication by Progressive Profiling. Journal of Food Science, 1994. 59(3): p. 539-543

[5] Pineau, N., et al., Temporal Dominance of Sensations: Construction of the TDS curves and comparison with time-intensity. Food Quality and Preference, 2009. 20(6): p. 450-455

[6] Labbe, D., et al., Temporal dominance of sensations and sensory profiling: A comparative study. Food Quality and Preference, 2009. 20(3): p. 216-221

[7] Methven, L., et al., The effect of consumption volume on profile and liking of oral nutritional supplements of varied sweetness: Sequential profiling and boredom tests. Food Quality and Preference, 2010. 21(8): p. 948-955

[8] Di Monaco, R., et al., Temporal Dominance of Sensations: A review. Trends in Food Science \& Technology, 2014. 38(2): p. 104-112

[9] Meillon, S., et al., Preference and acceptability of partially dealcoholized white and red wines by consumers and professionals. American journal of enology and viticulture, 2010. 61(1): p. 42-52

[10] Meillon, S., C. Urbano, and P. Schlich, Contribution of the Temporal Dominance of Sensations (TDS) method to the sensory description of subtle differences in partially dealcoholized red wines. Food Quality and Preference, 2009. 20(7): p. 490-499

[11] Meillon, S., et al., Impact of partial alcohol reduction in Syrah wine on perceived complexity and temporality of sensations and link with preference. Food Quality and Preference, 2010. 21(7): p. 732740

[12] Varela, P. and G. Ares, Sensory profiling, the blurred line between sensory and consumer science. A review of novel methods for product characterization. Food Research International, 2012. 48(2): p. 893-908

[13] Galmarini, M.V., et al., Understanding apple consumers' expectations in terms of likes and dislikes. Use of comment analysis in a cross-cultural study. Appetite, 2013. 62(0): p. 27-36

[14] Lenfant, F., et al., Perception of oral food breakdown. The concept of sensory trajectory. Appetite, 2009. 52(3): p. 659-667

[15] Monterymard, C., M. Visalli, and P. Schlich. The TDS-band plot: a new graphical tool for Temporal Dominance of Sensations data. in 2nd conference of the society of sensory professionals. 2010

[16] Sáenz-Navajas, M.-P., et al., Perception of wine quality according to extrinsic cues: The case of Burgundy wine consumers. Food Quality and Preference, 2013 27(1): p. 44-53

[17] Tu, V.P., et al., Cultural differences in food description and preference: Contrasting Vietnamese and French panellists on soy yogurts. Food Quality and Preference, 2010. 21(6): p. 602-610

[18] Valentin, D., S. Chollet, and H. Abdi, Les mots du vin: experts et novices diffèrent-ils quand ils décrivent des vins? Corpus, 2003(2) 